\title{
Modelling and Control of Unmanned Ground Vehicle
}

\author{
Zain Ul Abdin \\ Laboratoire des Technologies Innovantes \\ Université de Picardie Jules Verne \\ Amiens, France \\ zain.ul@u-picardie.fr
}

\author{
Taimur Islam Khan \\ National Electronics Complex of Pakistan \\ Islamabad, Pakistan \\ taimurislamkhan@gmail.com
}

\author{
Mazhar Shabir \\ Innovative Private Limited \\ Islamabad, Pakistan \\ mazharshabir1@gmail.com
}

\begin{abstract}
Unmanned Ground Vehicles (UGVs) are the next step to human safety by placing the human outside of the vehicle. They not only provide human safety but also have greater speed, mobility and can survive atmospheres that are lifethreatening to humans. The proposed vehicle has the capability of changing its ride height and traveling in rough terrains. In order to get the most precise structure and more precision, the following tests were performed for making the UGV optimum (i) sinkage which was done for heavy clay and sand, (ii) torque was tested on rubber and plane surface, (iii) projectile motion and impact force, (iv) diameter required for the shaft. Further, we identify an electromechanical transfer function connecting electrical input to mechanical output by finding equivalent inertia and damping. Finally, a simple PD controller is proposed to keep the actual output as close to the target as possible and proven to guarantee good performance. The simulation results show that the controller can satisfy tracking specifications.
\end{abstract}

Index Terms-UGV, modelling, identification, control

\section{INTRODUCTION}

Unmanned Ground Vehicles (UGVs) are developed to perform activities for both military and civilians. These vehicles not only provide human safety but are effective in a number of circumstances where human labor is expensive. Detailed mathematical modelling of all-terrain UGV obtained from various subsystems is presented by Dave and Patil [1]. A proportional-integral (PI) and proportional-integral-derivative (PID) controllers are designed using two different tuning techniques i.e. Ziegler Nichols open loop-set point tacking (ZNOL-SP), Ziegler-Nichols open loop-load distribution rejection (ZNOL-LDR) and applied to the non-linear model which is obtained by merging the subsystems. The simulated results indicate that the performance of PI controller is better than the PID controller even with the disturbance. It is further observed that the performance with ZNOL-SP PI controller is better than ZNOL-LDR PI controller. De Simone and Guida [2] identified a dynamic model of a UGV using numerical algorithms for subspace state-space system identification (N4SID). Further, open-loop and closed-loop control algorithms were executed using the Atmel Atmega 2560 controller. The obtained results showed the effectiveness of the proposed method. A detailed and complete dynamic model of Argo-J5 UGV by adding a vertical load on each wheel is presented by Alghanim et al. [3]. The vehicle modeling details include motor torque, wheel torque, vehicle-terrain interaction analysis, and vertical load distribution. A proportional-derivative (PD) controller is, then, designed to demonstrate the tracking capabilities of the vehicle. Moreover, the velocity, wheel rolling resistance, wheel turning moment resistance, and shear stress on different terrains of the vehicle are illustrated through simulations. Abdelhafid et al. [4] proposed the design of a remotely controlled teleoperated prototype UGV for security applications where several design configurations were developed and evaluated. Tran et al. [5] presented mathematical modelling of an autonomous amphibious vehicle developed at the Australian Centre for Field Robotics, Sydney. The driveline of the vehicle is divided into subsystems that include gearbox, differential (in a gearbox), chains, and wheels. The numerical results are compared to experimental ones in order to validate and demonstrate the viability and consistency of the proposed model. The obtained results show that these models can be used for control purposes. Haytham et al. [6] presented modelling of a four-wheel steering Unmanned Ground Vehicles. Moreover, PID controllers are designed and optimal genetic algorithm is developed to tune the controllers of vehicle. Experimental and simulated results showed that controller rapidly adjusts the heading of vehicle to follow the specified mission.

In this paper, a UGV is designed that is capable of moving through the following urban terrains: road surface, polished floor surface, grassy ground, sand, fine gravel, and mud (nonliquefied). Several mathematical tests were performed in order to get a more precise structure and precision. The major objective of this study is to identify an electromechanical model connecting electrical input to mechanical output by finding equivalent inertia and damping. Additionally, a PD controller is designed and proven to guarantee good performance.

The remainder of this paper is organized as follows: mathematical tests i.e. sinkage, torque, projectile motion, impact force, and diameter required for the shaft are presented in section II(A). In section II(B), an electromechanical model 


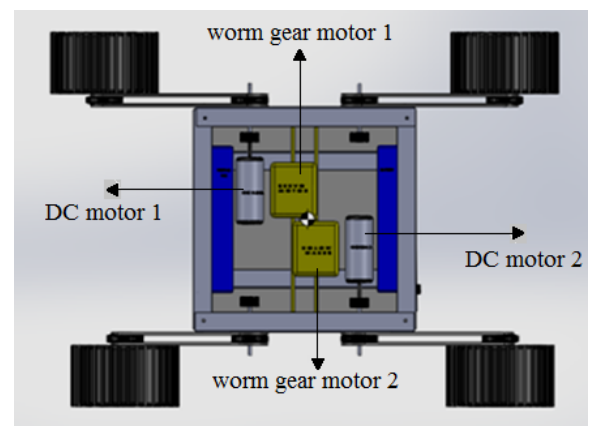

Fig. 1: Cross-sectional view of motors from top.

is developed that is a hybrid of electrical and mechanical variables and relates angular displacement and voltage. In section III, a simple PD controller is designed to ensure that the output reaches the desired setpoint. Finally, the paper ends with conclusions in section IV.

\section{Modelling}

\section{A. Mathematical Tests}

Modern Engineering is based on modelling and simulation and most of the manufacturing is based on this technology. Therefore, UGV's are generally based on mathematical tests in order to get the most precise structure and precision in particular. Following tests were performed for making the UGV optimum. These tests include sinkage which was done for heavy clay and sand. The exact tire width can be used in order to get avoid sink by finding the sinkage on different surfaces i.e. road surface, polished floor surface, grassy ground, sand, fine gravel, and mud (non-liquefied). Torque was tested on rubber and plane surface, motors give different values and by proving it mathematically required torque for each worm gear motor is approximately $0.98 \mathrm{Nm}$ whereas the torque required for DC motor is approximately $3 \mathrm{~N}$.m. Stress tests were done to find maximum shearing stress within the shaft and on the basis of the stress test, the diameter of the shaft is $0.48 \mathrm{~cm}$ with a safety factor of 1.5. Each panel including inner structure and tires were made in the 3D model shown in Fig. 8.

1) Sinkage: Sinkage is the measure of immersion into the surface on which the wheel is rolling or static after the weight is applied on it. The sinkage $Z$ can be written as [7]:

$$
Z=\sqrt[n]{\frac{P}{\frac{k_{c}}{b}+k_{\phi}}},
$$

where $P$ is ground pressure, $n$ is sinkage exponent, $b$ is the

\begin{tabular}{|c||c|c|c|c|c|}
\hline Surface & $P$ & $k_{c}$ & $k_{\phi}$ & $n$ & $Z(\mathrm{~cm})$ \\
\hline \hline Sand & 3034 & 990 & 1528430 & 1.1 & 0.347 \\
\hline Heavy Clay & 3034 & 12700 & 1555950 & 0.73 & 0.0178 \\
\hline
\end{tabular}

TABLE I: Sinkage for sand and heavy clay.

width of tyre, $k_{c}$ is cohesive modulus of soil deformation

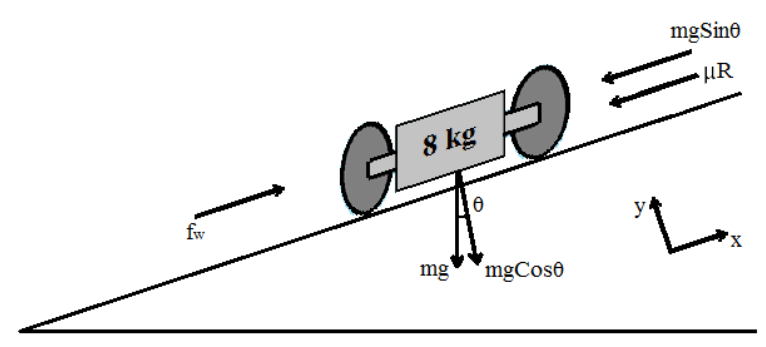

Fig. 2: Free body diagram of vehicle on inclined surface.

$\left(\mathrm{N} / \mathrm{m}^{2}\right)$, and $k_{\phi}$ is frictional modulus of soil deformation $\left(\mathrm{N} / \mathrm{m}^{3}\right)$. The ground pressure is respectively given by:

$$
P=\frac{F}{A}=\frac{m g}{4 W R},
$$

where $F$ is the load, $m$ is the mass of the UGV, $A$ is the area of tyre and is the product of width $W$ and radius $R$ of the tyre. Since, UGV contains four tyres so the area is multiplied by four times. The sinkage values for different surfaces are provided in Table 1. As the sinkage depth comes out to be very minor i.e. $0.0178-0.347 \mathrm{~cm}$ for different surfaces. It would be appropriate to use the tires with the above mentioned width.

2) Torque: In this section torque for DC motor (inclined and plane surface) and worm gear motor is calculated. There are two DC motors used in the vehicle, left DC motor controsls the left tires whereas the right DC motor controls the right tires. The height control motors produce moment which is transferred to the arms through a mechanism of sprockets and chains. The front two arms and the back two arms are connected to the worm gear height control motors as presented in Fig. 1.

- torque for DC motor (inclined surface):

$$
R=m g \operatorname{Cos} \theta
$$

$\theta$ is the angle between the force vector and the arm vector as shown in Fig. 2. The slope assumed for the inclined surface is $\theta=20^{\circ}$ and rolling resistance (rubber) $\mu=0.75$, force $f_{w}$ acting on the vehicle is written as [8]:

$$
f_{w}=\mu R+m g \operatorname{Sin} \theta
$$

Since, two DC motors are used so the torque $\tau$ for one motor can be written as:

$$
\tau=\frac{r f_{w}}{2},
$$

where $r$ is the vehicles position vector (a vector from the

\begin{tabular}{|c||c|}
\hline Surface & $\tau$ (N.m) \\
\hline \hline inclined surface & 3.13 \\
\hline plane surface & 2.99 \\
\hline
\end{tabular}

TABLE II: Torque for inclined and plane surface. 


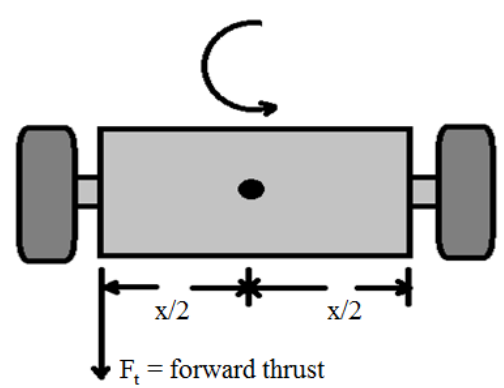

Fig. 3: Schematic diagram for height control motors.

point about which the torque is being measured to the point where the force is applied).

- torque for DC motor (plane surface):

$$
\tau=\frac{r F}{2},
$$

where $F$ is the force acting on the vehicle. The required torque for each motor is provided in Table 2 for both inclined and plane surfaces.

- worm gear DC motor for height control:

Torque $\tau$ by the worm gear motor is 0.9867 N.m and $F_{t}$ is the forward thrust as shown in Fig. 3.

$$
M_{t}=\frac{F_{t} x}{2},
$$

where $x$ is the width of the vehicle. The moment for a single motor is half of $M_{t}$ and is approximately equal to $3.43 \mathrm{~N} . \mathrm{m}$. This moment is enough to overcome the frictional force of tires and ground.

3) Impact force: An explorer UGV must be compliant with rough terrains which may encounter bumps and sometimes drops. Keeping this in mind, a limit is set to which the UGV can be dropped which is an average height of the stair as shown in Fig. 4. This will enable the UGV to travel at $2.3 \mathrm{~m} / \mathrm{s}$ with the mass of $8 \mathrm{~kg}$ and drop down on the ground forming a projectile motion. The UGV has its center of gravity in the direct center so it should theoretically follow a projectile motion.

- along y-axis

The time of flight can be calculated using

$$
-h=v_{i, y}-\frac{g t^{2}}{2},
$$

where vertical height $h$ is known, $v_{i, y}=0$ and the time of flight using (8) is $0.28 \mathrm{sec}$.

- along x-axis

Now by using time of flight, the distance covered along the $\mathrm{x}$-direction can be calculated by the following equation [8]:

$$
S_{x}=v_{f, x} t
$$

horizontal distance traveled during the flight time is $0.64 \mathrm{~m}$.

The impact force or the resulting force which will oppose the vehicle when it will jump from a certain height can be determined as follows:

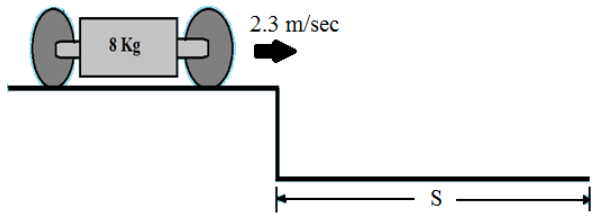

Fig. 4: Projectile motion.

The vertical velocity component $v_{f, y}$ is:

$$
v_{f, y}=\sqrt{2 g h},
$$

where $h$ is the height from where the vehicle is launched and is assumed as $0.381 \mathrm{~m}$. The horizontal velocity component $v_{f, x}$ is known and is $2.3 \mathrm{~m} / \mathrm{sec}$.

$$
v_{f}=\sqrt{v_{f, y}^{2}+v_{f, x}^{2}}
$$

Kinetic energy possessed by the object can be written as follows:

$$
K . E=\frac{1}{2} m v_{f}^{2},
$$

where the change in kinematic energy is the product of average impact force $F_{\text {avg }}$ and the total distance travelled $d$.

$$
F_{\text {avg }}=\frac{\frac{1}{2} m v_{f}^{2}}{d},
$$

where the distance travelled can be calculated using the equation given below.

$$
d=\sqrt{S_{x}+h^{2}}
$$

The $F_{\text {avg }}$ is $68.4 \mathrm{~N}$ with $d$ equal to $0.75 \mathrm{~m}$.

4) Diameter required for the shaft: The diameter required for the shaft with this much force to bear the shock when vehicle jumps from a certain height is calculated as follows:

The torque $\tau$ for one motor shaft is

$$
\tau=\frac{r F_{\text {avg }}}{2}
$$

The shear stress $T$ (for steel $430 \mathrm{MPa}$ ) can be written as [9]:

$$
T=\frac{\tau r}{J_{r}},
$$

where $r$ is radial distance from the center and can be expressed as $d_{r} / 2, J_{r}$ is polar second moment of area and can be expressed for a solid circular cross section as:

$$
J_{r}=\frac{\pi r^{4}}{2}=\frac{\pi\left(d_{r} / 2\right)^{4}}{2}
$$

(17) can be expressed as:

$$
d_{r}=\sqrt[3]{\frac{16 \tau}{\pi T}}
$$

Diameter of shaft $d_{r}$ is a critical component, so factor of safety is assumed as 1.5. The diameter required for the shafts is approximately equal to $0.48 \mathrm{~cm}$ and this diameter is enough to bear the shock when vehicle jumps from a certain height. 


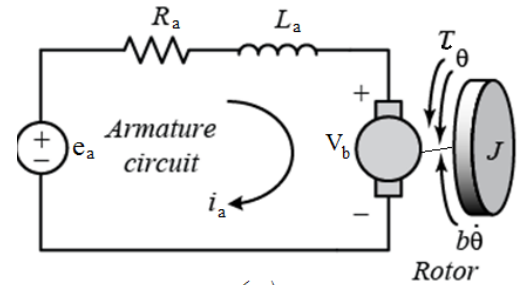

(a)

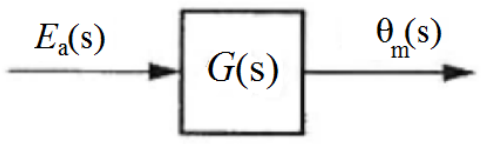

(b)

Fig. 5: DC motor (a) schematic, (b) block diagram.

\section{B. Electromechanical Identification}

In this part, the main purpose is to construct a model that approximates the behavior of a system using input and output data. Electromechanical transfer function connecting electrical input to mechanical output is determined by finding equivalent inertia and damping. The following equation is obtained by appling Kirchhoff's Voltage Law (KVL) [10] around the loop in clockwise direction in Fig. 5.

$$
R_{a} I_{a}(s)+L_{a} \frac{d I_{a}(s)}{d t}+V_{b}(s)=E_{a}(s),
$$

where $L_{a}$ is armature inductance, $R_{a}$ is armature resistance, $I_{a}$ is armature current, $V_{b}$ is the back electromotive force (emf) and the armature voltage $E_{a}$. Since the current-carrying armature is rotating in a magnetic field, its voltage is proportional to speed. Thus,

$$
V_{b}(s)=K_{b} \frac{d \theta_{m}(t)}{d t},
$$

where $K_{b}$ is a constant of proportionality called the back emf constant. By taking the Laplace transfor, yields

$$
V_{b}(s)=K_{b} s \theta_{m}(s)
$$

Torque developed by motor $T_{m}$ is proportional to the armature current: thus,

$$
T_{m}(s)=K_{t} I_{a}(s),
$$

where $K_{t}$ is constant of proportionality called motor torque constant. All the details for this section are provided in Appendix section. By rearranging (22), we get

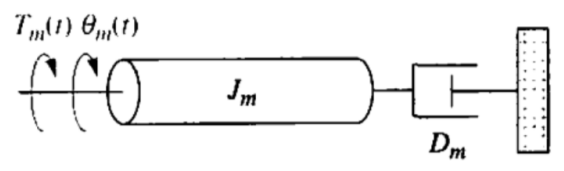

Fig. 6: Typical equivalent mechanical loading on a motor [10].

$$
I_{a}(s)=\frac{T_{m}(s)}{K_{t}}
$$

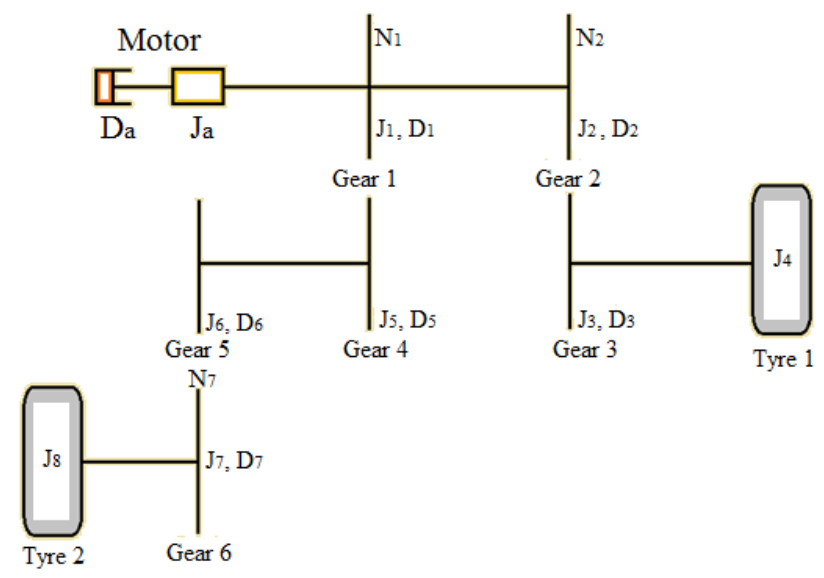

Fig. 7: DC motor driving a rotational mechanical load.

By substituting (20) and (22) in (19), we get

$$
\frac{\left(R_{a}+L_{a} s\right) T_{m}(s)}{K_{t}}+K_{b} s \theta_{m}(s)=E_{a}(s)
$$

Fig. 6 presents typical equivalent mechanical loading on a motor and $T_{m}$ can be written in terms of $\theta_{m}$;

$$
T_{m}(s)=\left(J_{m} s^{2}+D_{m} s\right) \theta_{m}(s),
$$

where $J_{m}$ is the equivalent inertia and $D_{m}$ is equivalent viscous damping at the armature. By assuming armature inductance $L_{a}$ to be small as compared to armature resistance $R_{a}$, so $L_{a}=0$. Now, substituting (25) in (24), yields

$$
\frac{R_{a}\left(J_{m} s^{2}+D_{m} s\right) \theta_{m}(s)}{K_{t}}+K_{b} s \theta_{m}(s)=E_{a}(s)
$$

Using (26), desired transfer function $\theta_{m} / E_{a}$ can be written as;

$$
\frac{\theta_{m}(s)}{E_{a}(s)}=\frac{K_{t} /\left(R_{a} J_{m}\right)}{s\left(s+\frac{1}{J_{m}}\left(D_{m}+\frac{K_{t} K_{b}}{R_{a}}\right)\right)}
$$

Now, consider Fig. 7, which shows a motor with inertia $J_{a}$ and damping $D_{a}$ at the armature driving a load consisting of inertia $\left(J_{1}-J_{8}\right)$ and damping $\left(D_{1}-D_{8}\right)$. The equivalent inertia, $J_{m}$, and equivalent damping, $D_{m}$, at the armature are

$$
\begin{aligned}
J_{m}=J_{a}+J_{1}+J_{2}+\left(J_{3}+J_{4}\right) & \left(\frac{N_{2}}{N_{3}}\right)^{2}+\left(J_{5}+J_{6}\right)\left(\frac{N_{1}}{N_{5}}\right)^{2} \\
+ & \left(J_{7}+J_{8}\right)\left(\frac{N_{1} N_{6}}{N_{5} N_{7}}\right)^{2}
\end{aligned}
$$

Similarly,

$$
\begin{array}{r}
D_{m}=D_{a}+D_{1}+D_{2}+\left(D_{3}+D_{4}\right)\left(\frac{N_{2}}{N_{3}}\right)^{2}+\left(D_{5}+D_{6}\right) \\
\left(\frac{N_{1}}{N_{5}}\right)^{2}+\left(D_{7}+D_{8}\right)\left(\frac{N_{1} N_{6}}{N_{5} N_{7}}\right)^{2}, \quad \text { (29) }
\end{array}
$$

where $N$ is the number of teeths of the gear, a gear with radius $r$ and teeth $N$ is rotated through angle $\theta$ due to a torque 


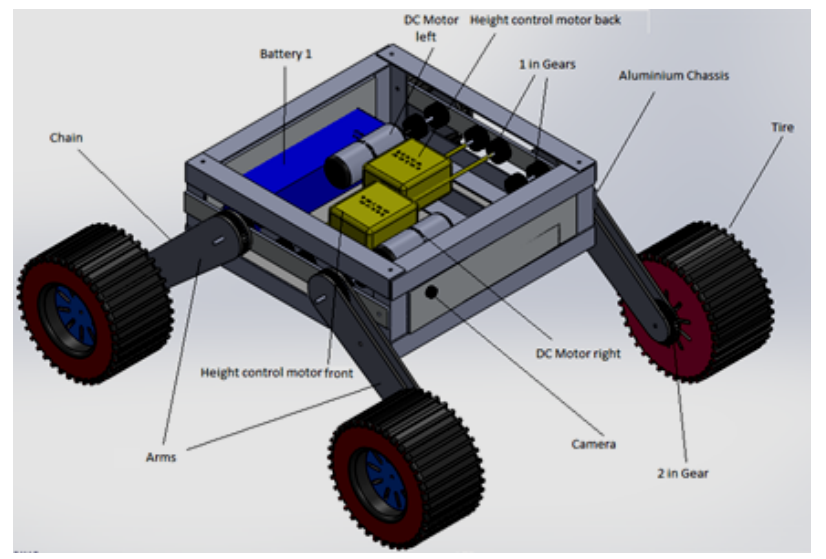

Fig. 8: Isometric view of the vehicle.

$\tau$. As the gears turn, the distance traveled along each gear's circumference is the same [10]. Thus, the rotation of two gears, yields

$$
\theta_{1} / \theta_{2}=N_{2} / N_{1}=r_{2} / r_{1}=\tau_{2} / \tau_{1}
$$

Thus, ratio of the angular displacement of the gears is inversely proportional to the torques. Where, the values for $N$ are $N_{1}$ $=15, N_{2}=30, N_{3}=30, N_{5}=15, N_{6}=30$ and $N_{7}=30$. Similarly, inertia's in $\mathrm{kg} \cdot \mathrm{m}^{2}$ are: $J_{a}=0.5, J_{1}=J_{5}=0.000125$, $J_{2}=J_{3}=J_{6}=J_{7}=0.001, J_{4}=J_{8}=0.0014$ and damping in N.m.s $/ \mathrm{rad}$ are $D_{a}=0.2, D_{1}=D_{5}=0.01, D_{2}=D_{3}=D_{6}=$ $D_{7}=0.008$. Now, the mechanical constants $J_{m}$ and $D_{m}$ are evaluted, the electrical constants are solved in the appendix section with $K_{t}=2.616 \mathrm{~N} . \mathrm{m} / \mathrm{A}$ and $K_{b}=2.952 \mathrm{~V} . \mathrm{s} / \mathrm{rad}$. The transfer function is found to be

$$
G(s)=\frac{\theta_{m}(s)}{E_{a}(s)}=\frac{0.16}{s(s+0.97)}
$$

The 3D model presented in Fig. 8 was designed using Solid Works 2013. The height control motors produce moment which is transferred to the arms through a mechanism of sprockets and chains.

\section{Controller Design}

In this section, the PD controller is designed and proven to guarantee good performance. The electromechanical transfer function obtained in (31) can be re-written as:

$$
G(s)=\frac{a}{s(s+p)}
$$

In terms of plant and controller parameters, the loop gain is

$$
G(s)=\frac{a\left(K_{d} s+K_{p}\right)}{s(s+p)}
$$

The closed-loop transfer function $F(s)$ from reference to output is

$$
F(s)=\frac{a K_{d} s+a K_{p}}{s^{2}+\left(p+a K_{d}\right) s+a K_{p}}
$$

This transfer function $F(s)$ denominator which is closed loop characteristic polynomial is compared with the second

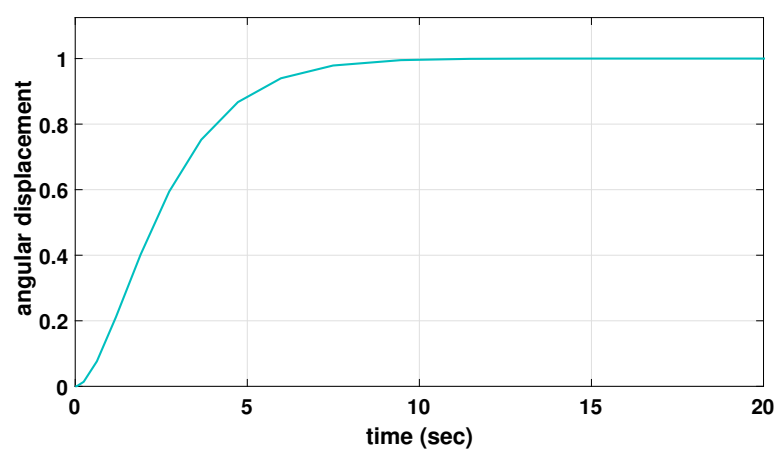

Fig. 9: Response of PD controller.

order general equation, assuming that desired characteristic polynomial is

$$
s^{2}+2 \zeta \omega_{n} s+\omega_{n}^{2}
$$

giving design equations

$$
\begin{gathered}
K_{d}=\frac{2 \zeta \omega_{n}-p}{a} \\
K_{p}=\frac{\omega_{n}^{2}}{a}
\end{gathered}
$$

The parameter $\omega_{n}$ determines the response speed while $\zeta$ relates to the damping [11]. The controller parameters are $K_{p}$ $=2.25$ and $K_{d}=0.69$ with $\zeta=0.9$ and $\omega_{n}$ is taken as 0.6 . It can be observed in Fig. 9 that the controller tracks the reference point.

\section{CONCLUSION}

In this study, several tests i.e. sinkage, the torque required, diameter needed for the shaft were performed. Sinkage depth comes out to be $0.0178 \mathrm{~cm}$ which is very small and it would be appropriate to use $0.085 \mathrm{~m}$ width of tires. The torque required by the motors is an important design parameter, influencing the performance of the vehicle. It was found that torque required by a single DC motor is approximately 3 N.m. The diameter required by the shaft is $0.48 \mathrm{~m}$ assuming a factor of safety of 1.5. Further, we have presented an electromechanical model of the vehicle connecting electrical input to mechanical output and its modelling is derived. A PD controller is developed for the proposed model to ensure that the output reaches the desired setpoint. Moreover, there is a need for physical experimentation of the vehicle to get a better knowledge of its feasibility and efficiency in real conditions.

\section{APPENDIX}

For (20), $d \theta_{m}(t) / d t=\omega_{m}(t)$ is the angular velocity of the motor. Taking the Laplace transform, we get

$$
\frac{d \theta_{m}(t)}{d t}=s \theta_{m}(s)
$$

Taking the inverse Laplace transform of (24) with $L_{a}=0$, yields

$$
\frac{R_{a} T_{m}(t)}{K_{t}}+K_{b} \omega_{m}(t)=E_{a}(t)
$$


by dropping the functional relationship based on time as motor will turn at a constant angular velocity with a constant torque. By solving above for $T_{m}$, we get

$$
T_{m}=-\frac{K_{b} K_{t}}{R_{a}} \omega_{m}+\frac{K_{t}}{R_{a}} E_{a}
$$

Using torque-speed curve, the torque axis intercept occurs when the angular velocity reaches zero and that is stall torque, Thus,

$$
T_{\text {stall }}=\frac{K_{t}}{R_{a}} E_{a}
$$

By solving above for $K_{t}$ we can calculate motor torque constant with $E_{a}=24 \mathrm{~V}, I_{a}=750 \mathrm{~mA}$ and $T_{\text {stall }}=20$ $\mathrm{kg} . \mathrm{cm}$. The angular velocity occurring when the torque is zero is called the noload speed, $\omega_{\text {no,load }}$, Thus,

$$
\omega_{n o, l o a d}=\frac{E_{a}}{K_{b}}
$$

By solving above for $K_{b}$ we can calculate back emf constant with $\omega_{\text {no,load }}=8.13 \mathrm{rad} / \mathrm{sec}$.

\section{ACKNOWLEDGMENT}

The authors would like to express very great appreciation to Amre Eizad for his valuable and constructive suggestions during the development of this research work.

\section{REFERENCES}

[1] P. N. Dave, and J. B. Patil. "Modeling and control of nonlinear unmanned ground all terrain vehicle," IEEE International Conference on Trends in Automation, Communications and Computing Technology (I-TACT-15), pp. 1-7, 2015.

[2] M. C. De Simone, and Domenico Guida, "Identification and control of a unmanned ground vehicle by using Arduino," UPB Sci. Bull. Ser., vol. D80, pp. 141-154, 2018.

[3] M. N. Alghanim, K. P. Valavanis, and M. J. Rutherford, "Modeling, control, and wheel-terrain interaction dynamics of the UGV argo J5," IEEE European Control Conference (ECC), 2019.

[4] A. Bouhraoua, N. Merah, M. AlDajani, and M. ElShafei, "Design and implementation of an unmanned ground vehicle for security applications," IEEE International Symposium on Mechatronics and its Applications, 2010.

[5] T. Tran, Q. P. Ha, R. Grover, and S. Scheding, "Modelling of an autonomous amphibious vehicle," Australasian Conference on Robotics and Automation, Australian Robotics and Automation Association (ARAA), 2004

[6] A. Haytham, Y. Z. Elhalwagy, A. Wassal, and N. M. Darwish, "Modeling and simulation of four-wheel steering unmanned ground vehicles using a PID controller," International Conference on Engineering and Technology (ICET), pp. 1-8, 2014.

[7] G. Pan, J. Sun, X. Wang, F. Yang, and Z. Liu, "Construction and Experimental Verification of Sloped Terrain Soil Pressure-Sinkage Model," Agriculture, vol. 11, p. 243, 2021.

[8] J. L. Meriam, L. G. Kraige, and J. N. Bolton, Engineering mechanics: dynamics, John Wiley \& Sons, 2020.

[9] F. Beer, E. Johnston, and J. DeWolf, Mechanics of materials, 5th SI Edition, Tata Mcgraw Hill, 2009.

[10] N. S. Nise, Control systems engineering, John Wiley \& Sons, 2020.

[11] Z. U. Abdin, and A. Rachid, "Modeling, Identification and Control of Photovoltaic/Thermal Solar Panel," IEEE Conference on Control Technology and Applications (CCTA), 2020. 